\section{PTH-054 FEASIBILITY OF NON-INVASIVE NASAL VENTILATION (THRIVE) FOR PROPOFOL-INDUCED SEDATION IN THERAPEUTIC UPPER GASTROINTESTINAL ENDOSCOPY}

${ }^{1}$ Nickki Pressler*, 'Zoe Riddell, ${ }^{2}$ Hamid Mohaghegh Shalmani, ${ }^{1}$ Keith Siau, ${ }^{1}$ Andrea Gait, ${ }^{1}$ Andrew Downs, ${ }^{3}$ Chris Mulder, 'Sauid Ishaq. 'Russell's Hall, Dudley, Birmingham, UK; ${ }^{2}$ Shahid Beheshti University of Medical Sciences, Tehran, Iran; ${ }^{3} \mathrm{VU}$ University Medical Center, Amsterdam, Netherlands

\subsection{6/gutjnl-2019-BSGAbstracts.79}

Background Pharyngeal conditions such as Zenker's Diverticulum (ZD) increase with age and may be amenable to endoscopic therapy. However, therapy is traditionally performed under general anaesthesia (GA) which requires ventilatory support and may preclude therapy in frailer patients. Transnasal Humidified Rapid-Insufflation Ventilatory Exchange (THRIVE) is a non-invasive nasal method of ventilation which obviates the need for tracheal intubation. However, evidence supporting the feasibility of THRIVE for in endoscopic procedures is lacking. This study aimed to assess the feasibility, efficacy and safety of THRIVE as an adjunct to deep sedation in endoscopic pharyngeal and upper oesophageal procedures.

Methods In this prospective study, consecutive patients undergoing therapeutic endoscopy of the pharynx and upper oesophagus between June 2016 and March 2018 were included. All procedures were performed under deep sedation with propofol and/or remifentanil in endoscopy unit as a day case. For $\mathrm{ZD}$, the pharyngeal pouch and the stomach were cleared of debris to reduce the risk of aspiration before sedation was up titrated to facilitate therapy. Sedation related adverse events and the total dose of sedative drugs used were recorded.

Results A total of 50 patients were included for analysis, with a mean age of 71.1 (range 31-93) and male patients comprising 58\%. 46\% were categorized as ASA grades I-II, 48\% Grade III and $6 \%$ as Grade IV. The median procedure time was 20 minutes. $83 \%$ of patients were sedated with both propofol (median dose $103 \mathrm{mg}$ ) and remifentanil (median dose $167 \mathrm{mcg}$ ) using a target controlled infusion under specialist anaesthetic supervision. THRIVE was commenced in all patients, with none requiring conversion to invasive ventilation. The commonest sedation related adverse event was transient hypotension (38\%) followed by bradycardia (8\%) and hypoxia $(8 \%)$. No procedures were abandoned due to complications. Patients achieved full post-procedure recovery from sedation after a median of 5 minutes.

Conclusions Deep sedation with THRIVE proved to be a useful and safe technique for advanced therapeutic upper GI endoscopy. The role of THRIVE may be generalisable to other invasive therapeutic modalities involving high-risk patients such as ERCP.

\section{PTH-055 BOUGIECAP DILATATION DEVICE: NOVEL ENDOSCOPIC METHOD FOR TREATMENT OF OESOPHAGEAL STRICTURES-RESULTS FROM A MULTICENTRE STUDY}

${ }^{1}$ Imdadur Rahman*, 'Philip Boger, 'Praful Patel, ${ }^{2}$ Benjamin Walter, ${ }^{2}$ Simone Schmidbaur, ${ }^{3}$ David Albers, ${ }^{3}$ Brigitte Schumacher, ${ }^{2}$ Alexander Meining. 'University Hospital Southampton NHS foundation trust, Birmingham, UK; ${ }^{2}$ UIm University Hospital, UIm, Germany; ${ }^{3}$ Elisabethkrankenhaus Hospital, Essen, germany

10.1136/gutjnl-2019-BSGAbstracts.80

Introduction Benign strictures in the upper GI tract are often treated endoscopically using Savary-Gillard bougie dilatators, which provide tactile feedback. However the drawback to this technique is the lack of direct optic feedback and the need for fluoroscopy during the procedure. A novel device, BougieCap (Ovesco, Germany), allows both tactile and optic feedback of the dilatation procedure without the need for fluoroscopy. The aim of this study was to assess the safety and efficacy of this device in a prospective cohort of patients. Methods Patients with benign strictures of the oesophagus and with clinical symptoms of dysphagia were recruited from 3 endoscopy centres in the UK and Germany for planned dilatation with the BougieCap. The device is a single use transparent conical cap which is fixed to the tip of the endoscope. It comes in different sizes to facilitate dilatation to varying diameters. Once in place, the endoscope is inserted and positioned in front of the stricture. Under direct vision, pushing forward and rotating with the endoscope enables the conical cap to dilate the mucosa in the area of the stricture by the conversion of longitudinal force into radial force vectors. Dilatation could be repeated sequentially with a larger sized cap if necessary. The primary outcome measure was technical success of dilatation. Secondary outcome measure was improvement in symptoms of dysphagia as assessed by the Dysphagia Handicap index (DHI) before and 14 day after the bougienage procedure and adverse events.

Results 79 patients (M/F 41/38) with benign oesophageal strictures underwent the procedure between February 2018 to January 2019. Aetiology of strictures were peptic 52\% ( $n=40)$, radiation $25 \%(\mathrm{n}=19)$, anastomotic $10 \%(\mathrm{n}=8)$, caustic $7 \%$ $(n=5)$, EoE 4\% $(n=3)$, post-ESD 3\% $(n=2)$. Mean diameter of strictures was $6 \mathrm{~mm}( \pm 2.9)$. Endoscopic bougienage was successful in $97.5 \%$. In 2 cases, with a narrow long stricture, bougienage failed because of high resistance at the site of the stricture causing buckling of the endoscope in the pharynx. Symptoms of dysphagia improved after bougienage (53.6 points. d0 v 26.4 points $\mathrm{d} 14, \mathrm{p}<0.01)$. Adverse events were loss of BougieCap in the stomach in 2 cases. No severe adverse events were reported.

Conclusions Endoscopic treatment of benign strictures using the BougieCap enables direct visual and tactile control of the bougienage procedure and therefore of mucosal damage within the area of strictures. This might help to adapt treatment even more precisely to the stricture. Symptoms of dysphagia are improved in short-term follow-up.

\section{PTH-056 MORTALITY \& READMISSION: A DESCRIPTIVE STUDY OF JAG ENDOSCOPY UNIT DATA}

1,2 Srivathsan Ravindran*, ${ }^{2}$ Hutan Ashrafian, ${ }^{3}$ Raphael Broughton, ${ }^{3}$ Michael Dron ${ }^{3}$ Tim Shaw, ${ }^{2}$ Ara Darzi, ${ }^{1,2,3}$ Siwan Thomas-Gibson. 'St Mark's Hospital, London; ${ }^{2}$ Department of Surgery and Cancer, Imperial College, London; ${ }^{3}$ The Joint Advisory Group on GI Endoscopy (JAG), London

\subsection{6/gutjnl-2019-BSGAbstracts.81}

Introduction As part of the JAG accreditation process, services are required to provide evidence that they monitor and act upon 30-day mortality (30-DM) and 8-day readmission (8-DR) following endoscopy. This data is currently not analysed or shared in any systematic way other than at unit level. The aim of this study was to gauge current trends across multiple centres, informing further work as part of the ISREE (Improving Safety and Reducing Error in Endoscopy) strategy.

Methods A retrospective analysis of JAG 30-DM and 8-DR evidence from 2013-18 was undertaken. Heterogeneous data 


\begin{tabular}{|c|c|c|c|c|}
\hline \multicolumn{5}{|c|}{ MORTALITY \& READMISSION OUTCOMES } \\
\hline \multirow{2}{*}{$\begin{array}{l}\text { Measure } \\
\text { Adjusted all-cause rates }\end{array}$} & \multicolumn{2}{|l|}{ 30-DM } & \multicolumn{2}{|l|}{ 8-DR } \\
\hline & Median & $0.61 \%$ & Median & $1.05 \%$ \\
\hline & Mean & $0.66 \%$ & Mean & $1.06 \%$ \\
\hline Adjusted endoscopy-related & Median & $0.00 \%$ & Median & $0.12 \%$ \\
\hline rates & Mean & $3.48 \%$ & Mean & $0.41 \%$ \\
\hline \multirow{6}{*}{$\begin{array}{l}\text { Procedures prior to mortality or } \\
\text { readmission (excluding unknown) }\end{array}$} & Upper GI (unspecified) & $71.63 \%$ & Lower GI (unspecified) & $44.52 \%$ \\
\hline & Lower GI (unspecified) & $16.23 \%$ & ERCP & $32.46 \%$ \\
\hline & ERCP & $9.55 \%$ & Upper GI (unspecified) & $21.27 \%$ \\
\hline & PEG & $2.01 \%$ & EUS & $1.75 \%$ \\
\hline & Mixed (upper \& lower) & $0.29 \%$ & & \\
\hline & EUS & $0.29 \%$ & & \\
\hline Endoscopy-related causes & Aspiration pneumonia & $20 \%$ & Abdominal pain & $28 \%$ \\
\hline \multirow[t]{13}{*}{ (excluding unknown) } & GI bleeding & $17 \%$ & Post-polypectomy & $17 \%$ \\
\hline & (therapeutic) & $14 \%$ & bleed & $12 \%$ \\
\hline & Pancreatitis & $14 \%$ & Bleeding (unspecified) & $9 \%$ \\
\hline & Perforation (therapeutic) & $10 \%$ & Pancreatitis & $8 \%$ \\
\hline & Other & $8 \%$ & Perforation & $8 \%$ \\
\hline & GI bleeding (diagnostic) & $6 \%$ & Other & $8 \%$ \\
\hline & Perforation (diagnostic) & $6 \%$ & Infection (unspecified) & $2 \%$ \\
\hline & Cholangitis & $3 \%$ & Sphincterotomy bleed & $2 \%$ \\
\hline & Sedation-related & $3 \%$ & Vomiting & $2 \%$ \\
\hline & Ischaemic heart disease & & Biopsy bleed & $2 \%$ \\
\hline & & & Bowel prep related & $1 \%$ \\
\hline & & & Chest pain & $1 \%$ \\
\hline & & & Sedation related & \\
\hline
\end{tabular}

was extracted from evidence files and collated to calculate mortality, readmission and procedure rates based on the minimum representative dataset. Causes related to endoscopy were identified through content analysis and grouped into appropriate headings. This study was approved by the JAG research committee.

Results In total, 501 data files were reviewed. Data was provided from 107 units and after exclusions, due to unusable and duplicate data, 51 units were included for analysis. Data covered 16,719 days of endoscopy and 281,566 endoscopic procedures. Outcomes from mortality and readmission data are summarised below:

Conclusions This study represents the most recent analysis of self-reported UK-wide data over a 5-year period. Adjusted allcause mortality rates appear similar to previously published literature. The commonest causes of endoscopy-related mortality and readmission were aspiration pneumonia and abdominal pain respectively. It is important to acknowledge these outcomes with a view to disseminate and share learning to improve future practice. These analyses will inform wider projects in improving safety in endoscopy.

\section{PTH-057 DISTRICT GENERAL HOSPITAL EXPERIENCE OF ENDOSCOPIC PAPILLARY LARGE BALLOON DILATATION VERSUS MORE CONVENTIONAL TECHNIQUES}

Matthew King, Lavan Velu, Laura Kelso, Kevin Robertson*. University Hospital Crosshouse, NHS Ayrshire and Arran, Glasgow, UK

10.1136/gutjnl-2019-BSGAbstracts.82
Introduction Endoscopic Papillary Balloon Dilatation (PBD) is a technique which can be used during ERCP to aid removal of common bile duct stones. An advancement of this technique, Endoscopic Papillary Large Balloon Dilation (PLBD), allows dilation of the lower bile duct up to $20 \mathrm{~mm}$ diameter. PLBD use has not been studied within a District General Hospital (DGH) setting and our study aimed to examine complications and efficacy after it was introduced to our DGH in May 2017.

Methods Data was collected prospectively from 969 consecutive ERCP procedures performed by a single operator between September 2014 and May 2018. Procedures for non-gallstone related pathology were excluded leaving 747 procedures. These were split into two groups; before PLBD (maximal papillary dilation $10 \mathrm{~mm}$ ) and after PLBD introduction (maximal papillary dilation $20 \mathrm{~mm}$.) Rates of complication (acute pancreatitis, perforation and bleeding), stent insertion and complete duct clearance were compared between groups. Chisquared tests were used for statistical analysis.

Results 534 procedures were undertaken before PLBD introduction and 213 after it. There was no difference in overall complication rates between the groups; 5.9\% (32 of 534) versus $5.1 \%$ (11 of 213), $p=0.6$. Before PLBD, 50 patients had dilation to $10 \mathrm{~mm}$ during their first ERCP. After PLBD introduction, 46 patients had dilation to a mean diameter of 15.5 $\mathrm{mm}$ (range 12-18 $\mathrm{mm}$ ). There was no difference in the complication rates between these groups; $10 \%$ (5 of 50) and $2.1 \%$ (1 of 46 ), $p=0.1$. However, use of PLBD was associated with a statistically significant improvement in complete bile duct clearance $85 \%$ (39 of 46 ) versus $66 \%$ (33 of 50 ), $p=0.03$ and a significant reduction in the need for pigtail 